\title{
An Optical Joint Angle Measurement Sensor based on an Optoelectronic Sensor for Robot Manipulators
}

\author{
Dalia Osman ${ }^{1)}$, Xinli $\mathrm{Du}^{1)}$, Wanlin $\mathrm{Li}^{2}$, Yohan $\mathrm{Noh}^{1 / 3)}$ \\ 1) Dept. of Mechanical and Aerospace Eng., Brunel University London, UB8 3PH, United Kingdom \\ 2) Centre for Advanced Robotics @ Queen Mary (ARQ), Queen Mary University London, E1 4NS, United Kingdom \\ 3) Dept. of Biomedical Eng., King's College London, London, SE1 7EH, United Kingdom
}

e-mail: dalia.osman@brunel.ac.uk,wanlin.li@qmul.ac.uk,xinli.du@brunel.ac.uk, yohan.noh@brunel.ac.uk (corresponding author)

\begin{abstract}
- this paper presents the design of a joint angle measurement sensor using optoelectronic reflective sensors for use with robotic applications. It offers a minimised solution to shape sensing that can easily be integrated into robotic joints. A mathematical model used to characterize sensor behaviour is presented, and validated experimentally. A single unit of a robotic jointed finger is designed, and the sensing principle is tested with rotational motion. Results are presented and compared to simulated values. While the mathematical model can accurately estimate output signal with varying displacements, limitations of angle range means an updated sensor model is required, with addition of real electrical properties such as saturation of signal and resistance of electrical components. Regression fitting method is therefore done and a third order function was able to accurately estimate the output signal over a simulated sweep of joint angle values.
\end{abstract}

Keywords-component; position sensing, optoelectronic sensor, optic technology, angle measurement sensor.

\section{INTRODUCTION}

Position sensing and shape sensing technologies have been utilised to perform closed-loop position or shape control for a great number of robot applications. For example, position sensing technologies are used to facilitate a robot arm to recognise its end-effector pose for manipulating tools in a factory, where a user can command the tool to move to a targeted position in the workspace [1]. Robot hands are designed to manipulate and grasp objects dexterously, and must be able to recognise precise position information of individual fingers in order to interact with the object with precision, without causing damage [2]. Recently, soft and flexible manipulators referred to as an underactuated system have been exploited in the medical field, and these manipulators have been utilised in minimally invasive surgery (MIS). Such a manipulator's shape is snake-like, facilitating them to be navigated through small openings or holes in the body, where conventional rigid link surgical instruments would find difficulty. Shape sensing has been adopted to perform close-loop shape control for such manipulators [3].

In general, a closed-loop position control system is implemented into robot applications using position or shape sensing technology. One typical position sensing approach is to use optical and magnetic encoders. Encoders with

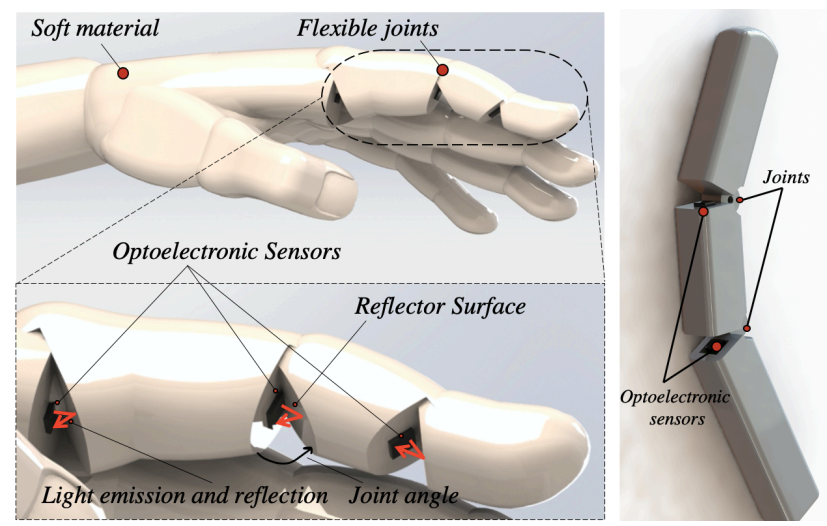

Figure 1. (Left) Concept soft robotic hand application to optoelectronic based joint angle measurement sensor (adapted from GrabCad, Joerg Schmit, 2012), (Right) designed protoype for initial experiments

actuators are integrated into each joint in a robotic application, measuring joint angles with high precision. An optical encoder consists of an LED, a photodiode (PD), and a rotational disk with a circumferential pattern of opaque lines or slits. As this rotates, light is transmitted through these slits in such a pattern from the LED to the PD, where it converts a high voltage to one (light received) and a low voltage to zero (blocked light). Through this binary signal counting (high to low) rotational angles can be calculated precisely [4]. A magnetic encoder consists of a hall sensor and a rotational disk magnetised with a patterned number of poles around its circumference. The hall sensor can detect magnetic flux density around the magnet poles. Through a certain pre-set threshold, the output of the hall sensor can generate a high voltage to one and a low voltage to zero. Based on counting the binary signals (high to low), rotational angles can be measured. The proportion of slit and magnet pole numbers on the disk pertains to an increase in the resolution of the encoders, although leads to an increase in size. In addition, fabricating a high-resolution disk with a large number of slits and poles results in higher cost [4].

Regarding robotic applications such as continuum flexible manipulators, for example robot hands actuated by a tendondriven mechanism, encoders would need to be installed externally, since the encoder with an actuator integrated directly at every joint in such a device is not dimensionally practical [5]. However in using actuation with antagonistic 
tendon mechanisms and tendon-driven mechanisms with a compression spring, an encoder installed remotely cannot measure precise joint angle data, since the tendon may be stretched by large tensions, or may be slack due to lack of pretension, causing position control in the tendon-driven mechanism to not guarantee precision[6] [7].

Such being the case, miniaturised position (angle) measurement sensors have been proposed by scientists that directly integrate into joints of robotic applications [5]. The article [8] shows an angle measurement sensor fabrication combining a hall sensor and a single bar magnet. The hall sensor measures magnetic flux intensity variations while the magnet is being rotated, converting them to voltage variations [9][8]. Through calibration voltages can be converted to physical quantities, and rotation angles can be estimated. Another two examples presented in the articles [10][11] introduce angle measurement sensors based on optoelectronic and fibre optic technology.

An LED as a light source and a photodiode (PD) as a detector are mounted facing each other at a joint of a robotic tendon actuated hand, and a variable-thickness canal installed between the two components can change light intensity gradually from the LED to the PD, eventually converting light intensity variations to physical quantities [11]. Another approach is to use the combination of multiple optical fibres, PDs, and an LED for angle measurement. The multiple optical fibres integrated into joints in a snake-like surgical robot are connected to the LED and PDs remotely. Using a customised mirror in the joint, light intensities are reflected and transmitted to the PDs, which can be used to estimate the angle information [10]. Other approaches have been proposed using a tiny rotary resistive sensor [12]. These were directly embedded into joints in a robotic finger application.

Although the mentioned optoelectronic technology approach may allow integrating an angle sensor into joints, it occupies a large space in the joint, resulting in an overall increased size of the robot application. The multiple optical fibres approach can not only measure a large range of joint angles, but also miniaturise the manipulator's diameter. Nevertheless, there would be difficulty in manufacturing the manipulator with the fibres, since in proportion to the number of links required, the total number of optical fibres integrated into the surgical instrument is increased [10]. Although optical fibres without a jacket can be installed to make the miniaturisation feasible, durability of this approach could be a crucial issue[13]. The small rotary resistive sensor approach has further miniaturization limitations of the overall size of rotary sensing system, despite the advantage of very simple integration into joints. Another issue is that the friction between the carbon film for its resistive track and sensor body arise; this mechanical friction including viscosity is unavoidable, causing short mechanical life [14].

In this paper, we are challenging the embodiment of a new rotary measurement sensor specialised in miniaturization, in simply being integrated into robot joints, and in fabricating it very simply at very low cost using an optoelectronic sensor.
This paper presents the advantage of an optoelectronic sensor on how it goes beyond the barrier of miniaturisation of the overall size of a robot, and describes the sensing principle of the sensor using light intensity modelling. Finally, a simple application embedding our proposed sensing system will be shown to verify its effectiveness.

\section{DESIGN CONCEPT}

\section{A. Design Requirement}

In this paper, the design requirement to the design of an angle measurement sensor includes:

1) The proposed angle measurment sensor can be simply integrated into any robot applications.

2) Optoelectronic sensors are utilised as a sensing element.

3) Large voltage variation without an amplifier and with low level noise.

4) Simple fabrication at considerably low cost in comparison with conventional angle measurement sensors using different sensing elelments as forementioned above.

To verify the effectiveness of the proposed design concept, a new angle measurement sensor is designed to implement into robot finger joints (Figure 1).

\section{B. Sensing principle of angle measurement with an optoelectronic sensor}

The sensing principle proposed for the joint angle measurement sensor is based on the intensity modulation of light. The used optoelectronic sensor (QRE1113 ON Semiconductor Reflective Sensor) is made up of an LED source, coupled with a phototransistor to detect light reflected off an opposing surface, with size $3.6 \times 2.9 \times 1.7 \mathrm{~mm}$. Depending on the distance of the reflective object, the light intensity variation collected by the detector varies in output voltage [15]. In implementing this principle into the finger joints of a robotic hand application, joint angles can be measured in one orientation of motion. The concept is shown in Figure 1, where the sensor is attached to the end of one

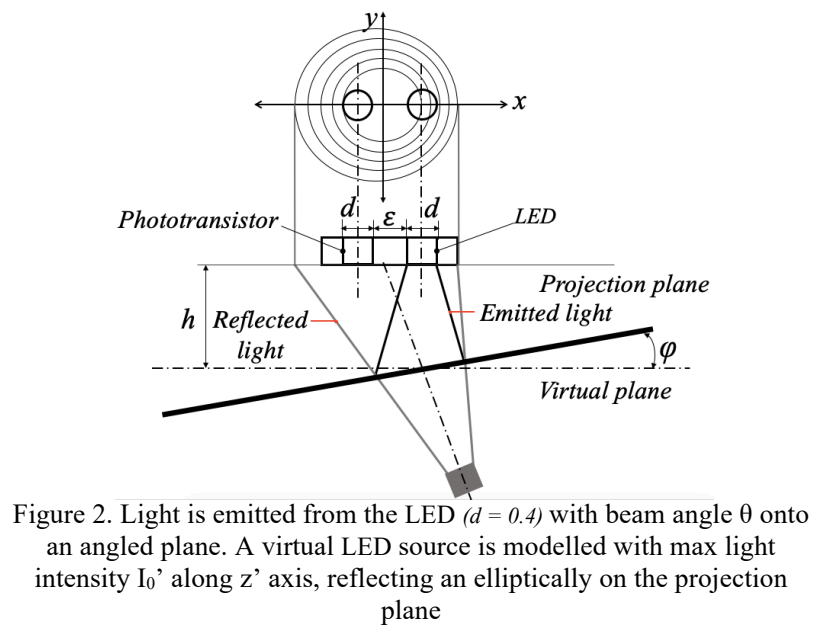


finger link, and while the next link rotates, it acts as a reflective surface, for which light intensity varies as it is reflected to the phototransistor. These voltage values can me mapped to real orientations, and used to estimate joint angle. This optoelectronic sensor is small, and can be directly embedded into such a joint, enabling the miniaturisation of the entire robotic structure. It is free from mechanical wear while also presenting a low cost alternative to the previous sensing approaches discussed [15][16]. Sensor signals are also resistant to ambient light if enclosed.

\section{Angle And Distance Measurement Principle}

\section{A. Light Intensity Model}

At first, it is important to characterise and understand the behaviour of the optoelectronic sensor being used. The sensor employed in this work is the QRE1113 Reflective sensor, which consists of an LED source and phototransistor detector. By modelling the proposed set up of the sensor geometrically, with an angled reflective surface, such as in Figure 2, a mathematical model can be derived to predict the output intensity level based on the amount of reflection. The figure describes the model with the various parameters, and assumes a gaussian light intensity distribution, with the reflecting surface set at initial distance $h$, and with variable angle $\varphi$ relative to the sensor plane, while the lateral distance between the sensor and the rotational axis of the surface is measured by $s$. The light emitted from the LED source has a Gaussian light intensity distribution. For a short range, it can be assumed that the light travels in a conically formed beam. Equation (1) shows this general relationship in Cartesian form;

$$
\mathrm{I}(\mathrm{x}, \mathrm{y})=\mathrm{I}_{0} \mathrm{e}^{-2\left(\frac{\mathrm{x}^{2}+\mathrm{y}^{2}}{\omega_{0}^{2}}\right)}
$$

where $I_{0}$ is the maximum intensity at the centre of the distribution, and $\omega_{0}$ is the mode field radius which is $1 / e$ of the peak intensity. This light is then reflected on the angled reflector surface and back to the projection plane. With the aid of a virtual diagram to map the path of the projection, it can be seen that the reflected light hits the projection plane with an elliptical cross section of this conical distribution due to angled planes. Hence, the light intensity emitted by the virtual LED source can be described by Equation (2):

$$
I(x, y)=I_{0}^{\prime} e^{-2\left(\frac{x^{2}}{a^{2}}+\frac{y^{2}}{b^{2}}\right)}
$$

where $I_{0}{ }^{\prime}$ is the new maximum intensity, with gaussian elliptical widths $a$ and $b$. Here, a symmetric distribution is assumed to simplify the model, as the non-centered elliptical distribution pattern can create complexities in solving for the flux [17]. The portion of light that is collected by the phototransistor is solved by calculating the total flux over the area of the phototransistor, represented by Equation (3), where $\phi_{c}$ is the collected flux, with the limits of integration found by geometrically finding the boundaries of the circular
LED and phototransistor structures. $a^{2}$ and $b^{2}$ represent the major and minor elliptical beam widths.

$$
\begin{aligned}
& \phi_{c} \\
& =\int_{h \tan (2 \varphi)-\frac{3}{2} d-\varepsilon}^{h \tan (2 \varphi)-\frac{1}{2} d-\varepsilon} \int_{-\sqrt{\left(\frac{d}{2}\right)^{2}-(y-h \tan (\theta)+d+\varepsilon)^{2}}}^{\sqrt{\left(\frac{d}{2}\right)^{2}-(y-h \tan (\theta)+d+\varepsilon)^{2}}} I_{0} e^{-2\left(\frac{x^{2}}{a^{2}}+\frac{y^{2}}{b^{2}}\right)} d y d x
\end{aligned}
$$

As the reflected light projects elliptically, these widths can be found by setting up an intersection plane onto a conical formula along the $z$, axis, solving for the equation of an ellipses, as described by equations (4) - (7) :

$$
\frac{x^{2}+y^{2}}{c^{2}}=m y+n^{2}
$$

Where:

$$
\begin{gathered}
c=\tan \theta \\
m=\frac{\sin 2 \varphi}{\cos 2 \varphi} \\
n=\frac{h}{\cos 2 \varphi}+h+\frac{d}{2 \tan \theta}
\end{gathered}
$$

Here shown is the equation of a cone with a substitution of the projection plane. $c$ represents the slope coefficient, which depends on beam angle $\theta . m$ represents the slope of the projection plane relative to the central beam axis $z$, while $n$ is its intersection with this axis. This is evaluated into the general form of an elliptical formula (8):

$$
\frac{\left(x-x_{c}\right)^{2}}{a^{2}}+\frac{\left(\mathrm{y}-y_{c}\right)^{2}}{b^{2}}=1
$$

This results in the values for $a$ and $b$ being calculated by:

$$
\begin{gathered}
a^{2}=c^{2} n^{2}+\frac{m^{2} n^{2} c^{4}}{1-c^{2} m^{2}} \\
b^{2}=\frac{a^{2}}{\cos (2 \varphi)\left(1-c^{2} m^{2}\right)}
\end{gathered}
$$

Once this (3) can be solved, the equation for the theoretical phototransistor signal voltage can then be formulated by Equation (11):

$$
V_{t h}=\phi_{c} \cdot R \cdot k_{v}
$$

where light losses are represented by reflection rate $\mathrm{R}$ and conversion coefficient is introduced by $k_{v}$, that converts the intensity into a voltage value. Depending on a particular application, it is beneficial to model the behaviour of such a sensor in terms of these factors as this would allow one to set design parameters for any potential device.

\section{B. Simulation Results}

Figure 3 shows the experimental set up used to validate the model. The sensor is fixed on one end of a motorized linear guide. A white PLA 3D printed reflector surface is coupled 


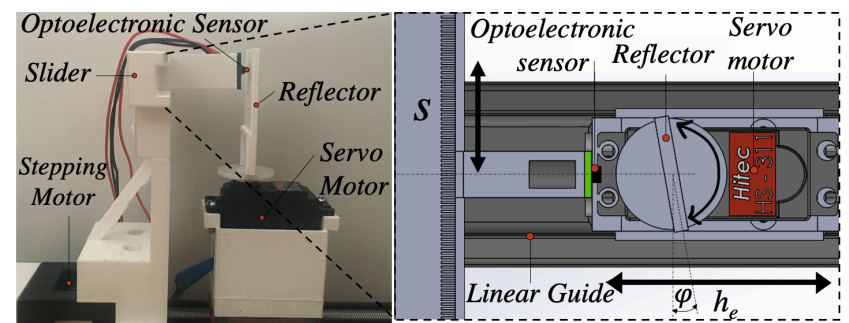

Figure 3. Experimental set up with linearly moving reflector, with rotation set by servo motor. Sensor is manually positioned in the lateral direction on a sliding mechanism
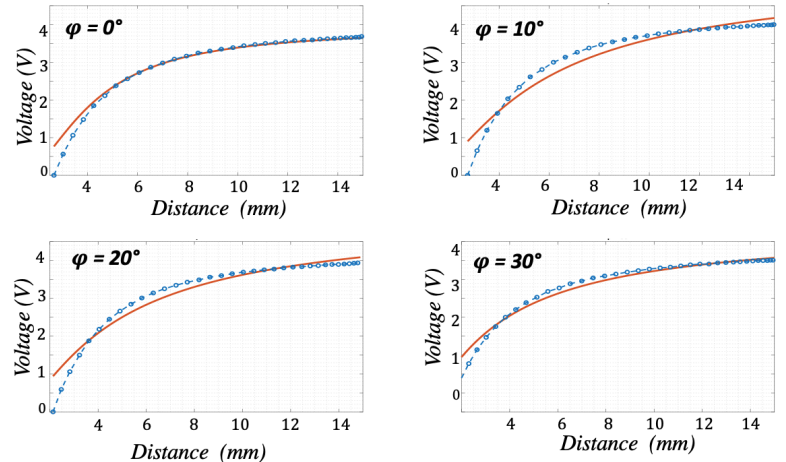

Figure 4. (a) Model simulated output voltage against displacement (orange) compared to experimentally collected data (blue) (parameters set to $s=0$, and $d=0.4 \mathrm{~mm}$ and $\theta=18^{\circ}$ )

Experimental vs Simulated data : (for $\left.\varphi=20^{\circ}\right)$
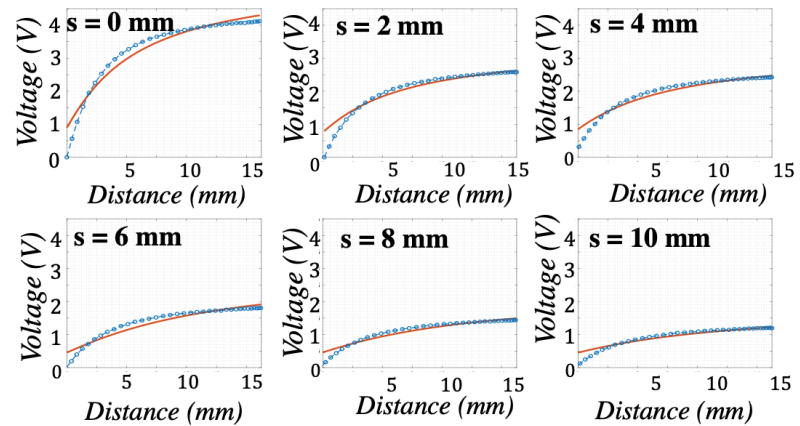

Figure 4. (b) Model simulated output voltage against displacement (orange) compared to experimentally collected data (blue), by varying the parameter $s$, to investigate sensor placement

to a servo motor that is mounted onto a sliding platform on the linear guide that allows it to rotate with angle $\varphi$. The reflective surface with the servo motor are able to slide longitudinally along the guide with varying distance $h_{e}$ from the fixed sensor. The sensor is mounted on the fixed end of the linear guide to a manual sliding attachment, that allows it to slide laterally from the central axis with distance $s$. For the collection of data, the servo motor rotates the reflective surface to form an angle with the sensor plane. Once fixed, the linear guide moves a programmed distance away from the sensor plane while a computer program simultaneously records the sensor voltage data at each displacement point. This data was compared to the theoretically simulated data calculated by Equation (11), in order to verify the model.
Figure 4 shows the results of the simulated and experimental voltage signals for these parameters.

\section{Embodiment of Angle Measurement SEnsor}

\section{A. Design of Prototype}

A simple finger prototype (Figure 5) consisting of two links with a single joint to allow rotational motion was designed. One link was grounded and the optoelectronic sensor was attached to the flat end surface. The second link was fixed to a motor horn attached to a servo motor, and the two links were joined with a screw along the central rotational axis of the motor. The end face of the rotating joint was used as a surface reflector, to reflect the light emitted from the opposing LED, to be measured by the adjacent phototransistor. The parts were designed on Solidworks and $3 \mathrm{D}$ printed. The motor was rotated continuously with a predefined angle range, while the sensor data was simultaneously recorded, using an Arduino Mega microcontroller board, to collect data into an excel file. Experimental results are displayed in Figure 6 and 7.

\section{ANALYSIS}

The results shown in Figure 4 validate the mathematical model derived in Section III, where different sensor and reflector configurations were set up experimentally, specifically testing different surface angles with varying displacements away from the sensor. When these same parameters are inputted into the mathematical equations to estimate output and compared to the experimental data, it can be seen that the curve fits the data closely, with RMS errors for data in Figure 4(a)(b) shown in Table 1. Figure 4(b) highlights the effect of the placement of the sensor away from the rotational axis, as this was considered when designing a prototype (Figure 5), and is useful in identifying a sensing range for a required application. Figure 6 compares the simulated output of continually varying the reflector angle relative to the optoelectronic sensor, based on the model. Although it appears at first to generally follow the tendency of the measured experimental data, the output voltage appears to drop after a certain angle. This can be explained theoretically, in that after a certain angular limit, the infrared light entering the phototransistor begins to reduce, as the beam can no longer be projected on the sensor plane. This presents itself as a drop in voltage in the simulated data. In

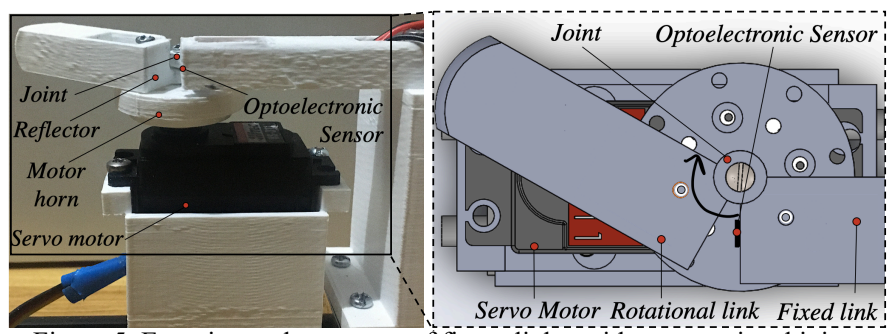

Figure 5: Experimental prototype of finger links, with one rotational joint, coupling to a servo motor to allow rotational motion to carry out angular tests 


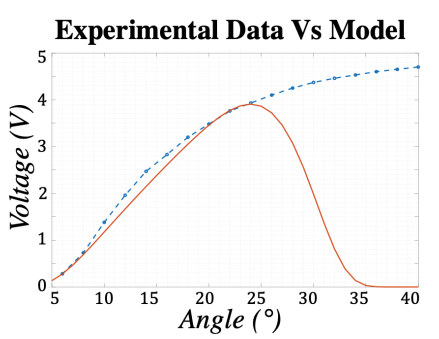

Figure 6. Comparison of experimental data (blue) with varying reflector rotation compared with simulated output signal

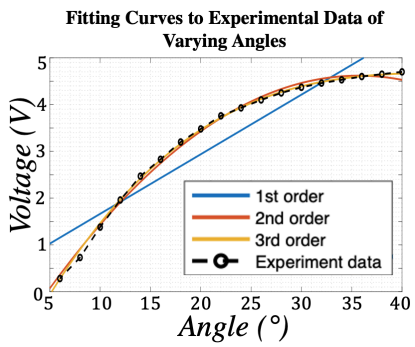

Figure 7. Experimental data collected with varying reflector angle, compared to data fitting reality, when this happens, the sensor measures a saturated output voltage equal to the supply voltage, as the light levels entering the transistor are so low that change in voltage is not seen. This property is not reproduced in the mathematical equations describing the model, hence it is not able to fully describe the sensor output behaviour with varying reflector angles. In future work these effects should be incorporated to provide a better estimation of the characteristic behaviour. For this reason, another approach was taken to find a polynomial function to fit the data as in figure 7 , where $1^{\text {st }}$, $2^{\text {nd }}$ and $3^{\text {rd }}$ order functions are fitted. It can be seen here that the $3^{\text {rd }}$ order function closely fits the experimental data, using least square based methods and can be used to map the output voltage values to the corresponding joint angle. The sensing range of the joint angles is typically between $0^{\circ}-40^{\circ}$. Future proposals may include considering different surface properties to increase the sensing range for application as a joint angle measurement sensor. For practical application, a cover should be designed to block noise due to external light.

\section{CONCLUSION}

In this work a simple integration of an optoelectronic sensor for joint angle measurement sensing has been proposed, for use in robotic applications. A light model has been derived to describe the characteristic behaviour of the reflective sensor, in order to be able to design for a tailored sensing unit. While this model was able to accurately estimate the behaviour with increasing displacements of a sensor from a reflector compared to experimental results, this was not the case when modelling over a sweep of angular rotations. To improve this outcome, the mathematical model should include features such as signal saturation and resistor values of the LED and phototransistor, in order to simulate more realistic conditions. Different surface properties for increased sensing range, as well as effects of vibration during motion will be investigated. This sensing scheme is suitable for applications in robotics, as its miniature, low cost qualities make it widely accessible, plus the sensors do not suffer from mechanical wear, and can be further miniaturised, by using for example a tiny sensor such as NJL5901R-2 (New Japan Radio). Following the suggested improvements, the testing of a robotic hand with further joints will be continued.
Table 1. RMS error values between simulated and experimental data for varying surface angle $(\varphi)$ and sensor placement (s) from rotational axis.

\begin{tabular}{|c|c|}
\hline $\begin{array}{c}\text { Surface angle } \\
(\varphi)\end{array}$ & RMS error \\
\hline 0 & 0.0053 \\
\hline 10 & 0.0081 \\
\hline 20 & 0.0081 \\
\hline 30 & 0.0074 \\
\hline
\end{tabular}

\begin{tabular}{|c|c|}
\hline s distance (mm) & RMS error \\
\hline 0 & 0.0084 \\
\hline 2 & 0.0061 \\
\hline 4 & 0.0058 \\
\hline 6 & 0.0046 \\
\hline 8 & 0.0037 \\
\hline 10 & 0.0036 \\
\hline
\end{tabular}

\section{REFERENCES}

[1] R. Li, H. Qiao, "A Survey of Methods and Strategies for HighPrecision Robotic Grasping and Assembly Tasks-Some New Trends,"IEEE/ASMETrans.Mechatronics,vol.24, no.6,pp.2718-2732, 2019.

[2] T. Mańkowski, J. Tomczyński, K. Walas, and D. Belter, "Puthand-hybrid industrial and biomimetic gripper for elastic object manipulation," Electron., vol. 9, no. 7, pp. 1-26, 2020.

[3] C. Shi et al., "Shape sensing techniques for continuum robots in minimally invasive surgery: A survey," IEEE Trans. Biomed. Eng., vol. 64, no. 8, pp. 1665-1678, 2017.

[4] S. Mathis, "Resolution, accuracy and precision of encoders," Assembly, vol. 62, no. 10, p. 2020, 2019.

[5] E. Mattar, "A survey of bio-inspired robotics hands implementation: New directions in dexterous manipulation," Rob. Auton. Syst., vol. 61, no. 5, pp. 517-544, 2013.

[6] B. Lukic, K. Jovanovic, and T. B. Sekara, "Cascade Control of Antagonistic VSA-An Engineering Control Approach to a Bioinspired Robot Actuator," Front.Neurorobot., vol.13, no. September, pp. 1-15, 2019.

[7] D. G. Lee and T. W. Seo, "Lightweight multi-DOF manipulator with wire-driven gravity compensation mechanism," IEEE/ASME Trans. Mechatronics, vol. 22, no. 3, pp. 1308-1314, 2017.

[8] Y. Park and J. Bae, "A three-dimensional finger motion measurement system of a thumb and an index finger without a calibration process," Sensors (Switzerland), vol. 20, no. 3, 2020.

[9] P. Agarwal, J. Fox, Y. Yun, M. K. O’Malley, and A. D. Deshpande, "An index finger exoskeleton with series elastic actuation for rehabilitation: Design, control and performance characterization," Int. J. Rob. Res., vol.34, no.14, pp.1747-1772, 2015.

[10] A. Schmitz, A. J. Thompson, P. Berthet-Rayne, C. A. Seneci, P. Wisanuvej, and G. Z. Yang, "Shape sensing of miniature snake-like robots using optical fibers," IEEE Int. Conf. Intell. Robot. Syst., vol. 2017-Septe, pp. 947-952, 2017.

[11] G. Palli and S. Pirozzi, "An optical joint position sensor for anthropomorphic robot hands,"Proc.-IEEE Int.Conf.Robot. Autom., pp. 2765-2770, 2013.

[12] A. Othman, N. Hamzah, Z. Hussain, R. Baharudin, A. D. Rosli, and A. I. C. Ani, "Design and development of an adjustable angle sensor based on rotary potentiometer for measuring finger flexion," Proc. 6th IEEE Int. Conf. Control Syst. Comput. Eng. ICCSCE 2016, no. November, pp. 569-574, 2017.

[13] S. Addanki, I. S. Amiri, and P. Yupapin, "Review of optical fibersintroduction and applications in fiber lasers," Results Phys., vol. 10, no. June, pp. 743-750, 2018.

[14] C. Michael, "BuRnishing and aDHESIVE WEAR of AN ELECtRically CONDUCTIVE POLYESTER-CARBON” vol. 132, pp. 265-285, 1998.

[15] Y. Noh, S. Han, P. Gawenda, W. Li, S. Sareh, and K. Rhode, "A Contact Force Sensor Based on S-Shaped Beams and Optoelectronic Sensors for Flexible Manipulators for Minimally Invasive Surgery (MIS)," IEEE Sens.J., vol. 20, no. 7, pp. 3487-3495, 2020.

[16] J. H. Benjamin Koh, T. Jeong, S. Han, W. Li, K. Rhode, and Y. Noh, "Optoelectronic Sensor-based Shape Sensing Approach for Flexible Manipulators," Proc. Annu. Int. Conf. IEEE Eng. Med. Biol. Soc. EMBS, pp. 3199-3203, 2019.

[17] P. Polygerinos, L. D. Seneviratne, and K. Althoefer, "Modeling of light intensity-modulated fiber-optic displacement sensors," IEEE Trans. Instrum. Meas., vol. 60, no. 4, pp. 1408-1415, 2011. 